\title{
First-order dipolar phase transition in the Dicke model with infinitely coordinated frustrating interaction
}

\author{
S. I. Mukhin ${ }^{1,2}$ and N. V. Gnezdilov ${ }^{1}$ \\ ${ }^{1}$ Instituut-Lorentz, Universiteit Leiden, P.O. Box 9506, 2300 RA Leiden, The Netherlands \\ ${ }^{2}$ Theoretical Physics and Quantum Technologies Department, National University of Science and Technology "MISIS," \\ Leninski Avenue 4, 119991 Moscow, Russia
}

(Received 12 February 2018; published 7 May 2018)

\begin{abstract}
We found analytically a first-order quantum phase transition in a Cooper pair box array of $N$ low-capacitance Josephson junctions capacitively coupled to resonant photons in a microwave cavity. The Hamiltonian of the system maps on the extended Dicke Hamiltonian of $N$ spins $1 / 2$ with infinitely coordinated antiferromagnetic (frustrating) interaction. This interaction arises from the gauge-invariant coupling of the Josephson-junction phases to the vector potential of the resonant photons field. In the $N \gg 1$ semiclassical limit, we found a critical coupling at which the ground state of the system switches to one with a net collective electric dipole moment of the Cooper pair boxes coupled to a super-radiant equilibrium photonic condensate. This phase transition changes from the first to second order if the frustrating interaction is switched off. A self-consistently "rotating" Holstein-Primakoff representation for the Cartesian components of the total superspin is proposed, that enables one to trace both the first- and the second-order quantum phase transitions in the extended and standard Dicke models, respectively.
\end{abstract}

DOI: 10.1103/PhysRevA.97.053809

\section{INTRODUCTION}

Realization of the equilibrium photonic condensates is of great interest for a fundamental study of new states of light strongly coupled to quantum metamaterials [1-6]. In particular, quantum electrodynamics of superconducting qubits in a cavity is crucial for the quantum computation perspectives [7-10]. In quantum optics (see, e.g., cavity QED described by the famous Dicke model [11]), the "no-go" theorems made perspectives of equilibrium photonic condensates gloomy [12-14], and only dynamically driven condensates are considered [15-20]. Nevertheless, it was found that in the equilibrium circuit QED systems the no-go theorems may not hold [4,5]. In particular, an array of capacitively coupled Cooper pair boxes to a resonant cavity was proven to disobey the no-go theorem for an equilibrium super-radiant quantum phase transition [5]. Nevertheless, another complication was found in this case, i.e., it was demonstrated $[6,21,22]$ that allowance for the gauge invariance with respect to the electromagnetic vector potential of the photon field causes the Hamiltonian of the system to map on the extended Dicke model Hamiltonian of (pseudo)spins $1 / 2$, adding to the standard Dicke model a frustrating infinitely coordinated antiferromagnetic interaction between the spins. Lately, numerical diagonalization results for small clusters with $N$ spins were reported [6] to behave differently depending on the parity of the number of spins $N$.

Motivated by the above history of exploration of the extended Dicke model, we present in this paper analytic description of the super-radiant equilibrium quantum phase transition in the array of $N \gg 1$ Cooper pair boxes strongly coupled to a resonant cavity. The plan of the present paper is as follows.

First, we reproduce derivation [6,21] of the extended Dicke Hamiltonian with an infinitely coordinated antiferromagnetic (frustrating) term. Next, we confirm the absence of the zero modes in the spectrum of the bosonic excitations, as was found in [6]. Then, we introduce a representation for the operators of Cartesian components of the total spin ("superspin") of $N$ spins 1/2: a self-consistently rotating Holstein-Primakoff (RHP) representation. After that, we demonstrate that RHP method applied to the extended Dicke Hamiltonian reveals the first-order quantum phase transition, that sets the system into a double degenerate dipolar ordered super-radiant state with a coherent photonic condensate emerging in the cavity. Also, in Appendix B we show that the RHP approach also reproduces the second-order quantum phase transition for the Dicke Hamiltonian without the frustrating interaction term, found earlier by another method [23,24]. We discuss a drastic difference between the critical values of the coupling strength $g_{c}$ in the $N \rightarrow \infty$ limit for the first- and second-order phase transitions, respectively. In the Conclusions we present some evaluations of the parameters of a Cooper pair boxes array in a microwave cavity for an experimental validation of our theoretical predictions.

\section{DICKE HAMILTONIAN OF A COOPER PAIR BOXES ARRAY}

In this section we present a derivation of the extended Dicke model Hamiltonian. We consider a single mode electromagnetic resonant cavity of a linear dimension $L$ coupled to an array of $N$ independent dissipationless Josephson junctions (JJs). It is assumed that the wavelength $\lambda$ of the cavity's resonant photon is much greater than the interjunction distance: $\lambda \gg L / N$. The vector potential of the electromagnetic field related with the photon is expressed in the second quantized form

$$
\vec{A}=\sqrt{\frac{c^{2} h}{\omega V}}\left(\hat{a}^{\dagger}+\hat{a}\right) \vec{\epsilon}
$$


where $h$ is Planck's constant, $\omega$ is the bare photon frequency, the photon creation and annihilation bosonic operators are $\hat{a}^{\dagger}$ and $\hat{a}, \vec{\epsilon}$ is a unit vector of polarization of electric field, $c$ is velocity of light, and $V$ is the volume of a cavity.

The Hamiltonian of a Cooper pair boxes array in a cavity then reads

$$
\begin{gathered}
\hat{H}=\hat{H}_{\mathrm{ph}}+\hat{H}_{\mathrm{JJ}}, \\
\hat{H}_{\mathrm{ph}}=\frac{1}{2}\left(\hat{p}^{2}+\omega^{2} \hat{q}^{2}\right), \\
\hat{H}_{\mathrm{JJ}}=E_{C} \sum_{i=1}^{N} \hat{n}_{i}^{2}-E_{J} \sum_{i=1}^{N} \cos \left(\hat{\phi}_{i}-\frac{g}{\hbar} \hat{q}\right),
\end{gathered}
$$

where the coupling constant is $g=2 e l \sqrt{4 \pi} / \sqrt{V}$, and $l$ is of the order of a penetration depth of electric field into the superconducting islands constituting a given Josephson junction, thus giving an effective thickness across the junction [22]. For simplicity, we consider all the junctions being identical, with electric-field polarization $\vec{\epsilon}$ aligned across each Josephson junction. Here the two mutually commuting sets of conjugate variables are introduced: $[\hat{p}, \hat{q}]=-i \hbar$ and $\left[\hat{n}_{i}, \hat{\phi}_{i}\right]=$ $-i$. The second quantized (harmonic oscillator) variables of the photonic field are

$$
\hat{p}=i \sqrt{\frac{\hbar \omega}{2}}\left(\hat{a}^{\dagger}-\hat{a}\right) \text { and } \hat{q}=\sqrt{\frac{\hbar}{2 \omega}}\left(\hat{a}^{\dagger}+\hat{a}\right),
$$

where $\left[\hat{a}, \hat{a}^{\dagger}\right]=1$. An operator $2 e \hat{n}_{i}=2 e\left(\hat{n}_{i}^{R}-\hat{n}_{i}^{L}\right) / 2$ stands for half of the charge difference at the $i$ th junction, and equals half the difference of the number of Cooper pairs populating left and right islands of the Josephson junction accordingly, multiplied by the elementary charge $2 e$ of the Cooper pair. The quantum of charging energy of a single junction is $E_{C}=$ $(2 e)^{2} / 2 C$.

Following [21] we make a canonical transformation:

$$
\hat{\phi}_{i}^{\prime}=\hat{\phi}_{i}-\frac{g}{\hbar} \hat{q} \quad \text { and } \quad \hat{n}_{i}^{\prime}=\hat{n}_{i}
$$

for the JJ variables and

$$
\hat{p}^{\prime}=\hat{p}+g \sum_{i=1}^{N} \hat{n}_{i} \quad \text { and } \quad \hat{q}^{\prime}=\hat{q}
$$

for photonic variables, so that $\left[\hat{p}^{\prime}, \hat{\phi}_{i}^{\prime}\right]=0$ and the other commutation relations between all the operators remain intact. The Hamiltonian (2) becomes

$$
\begin{aligned}
\hat{H}= & \frac{1}{2}\left(\hat{p}^{2}+\omega^{2} \hat{q}^{2}\right)-g \hat{p} \sum_{i=1}^{N} \hat{n}_{i}+\frac{g^{2}}{2}\left(\sum_{i=1}^{N} \hat{n}_{i}\right)^{2} \\
& +\sum_{i=1}^{N}\left(E_{C} \hat{n}_{i}^{2}-E_{J} \cos \hat{\phi}_{i}\right),
\end{aligned}
$$

where primes identifying the new variables are omitted for brevity. Thus, the infinitely coordinated interaction term $\propto g^{2}$ has appeared in (8) after the canonical transformation of the Hamiltonian (2).

We restrict ourselves to the Cooper pair box limit [25], when charging energy $E_{C}$ is large in comparison with Josephson coupling $E_{J}$ and the eigenstates of the Hamiltonian (4) in the zeroth-order approximation can be chosen as the eigenstates of the charge difference operators $\hat{n}_{i}$. The lowest bare energy level corresponding to the quantum states $\left|n_{i}=-\frac{1}{2}\right\rangle$ and $\left|n_{i}=\frac{1}{2}\right\rangle$ is thus twofold degenerate with respect to the direction of a Cooper pair box dipole moment $\vec{d}_{i}=2 e l \hat{n}_{i} \vec{\epsilon}$ ( $l$ is effective thickness of the $i$ th $\mathrm{JJ})$. This double degenerate level is separated from the levels with the greater charge differences by the $E_{C}$ gap. The Josephson tunneling term $\sim E_{J}$ lifts the degeneracy and opens a gap between the energy levels of the two states that differ by the wave-function parity \pm 1 with respect to inversion of dipole direction of the Cooper pair box. The thus formed two-level system is naturally described by the Pauli matrices $\hat{\sigma}_{i}^{\alpha}$. On the subset of these lowest-energy states the initial Hamiltonian (4) of the array of $N$ Josephson junctions that couple Cooper pair boxes is represented by a Hamiltonian of $N$ interacting spins $1 / 2$ :

$$
\begin{aligned}
\hat{H}_{\mathrm{JJ}}= & \sum_{i=1}^{N}\left(E_{C} \hat{n}_{i}^{2}-E_{J} \cos \hat{\phi}_{i}\right) \\
\approx & \frac{E_{C}}{4} \sum_{i=1}^{N}\left(\left|\frac{1}{2}\right\rangle\left\langle\frac{1}{2}|+|-\frac{1}{2}\right\rangle\left\langle-\frac{1}{2}\right|\right)_{i} \\
& -\frac{E_{J}}{2} \sum_{i=1}^{N}\left(\left|\frac{1}{2}\right\rangle\left\langle-\frac{1}{2}|+|-\frac{1}{2}\right\rangle\left\langle\frac{1}{2}\right|\right)_{i} \\
= & \frac{N E_{C}}{4} \hat{1}-\frac{E_{J}}{2} \sum_{i=1}^{N} \hat{\sigma}_{i}^{x} .
\end{aligned}
$$

Here charge and phase difference operators $\hat{n}_{i}$ and $\cos \hat{\phi}_{i}$ are projected on $\hat{s}_{i}^{z}$ and $\hat{s}_{i}^{x}$ correspondingly, where $\hat{s}_{i}^{\alpha}=\frac{1}{2} \hat{\sigma}_{i}^{\alpha}$ are spin-1/2 operators expressed via the Pauli matrices. As a result, initial Hamiltonian (8) reduces to the following spin-boson Hamiltonian, modulo energy shift $N E_{C} / 4$ :

$$
\begin{aligned}
\hat{H}= & \frac{1}{2}\left(\hat{p}^{2}+\omega^{2} \hat{q}^{2}\right)-g \hat{p} \sum_{i=1}^{N} \hat{s}_{i}^{z} \\
& -E_{J} \sum_{i=1}^{N} \hat{s}_{i}^{x}+\frac{g^{2}}{2}\left(\sum_{i=1}^{N} \hat{s}_{i}^{z}\right)^{2} .
\end{aligned}
$$

It is important to clarify here the meaning of the spin-boson interaction term in (10), that had emerged when canonical transformation (6) and (7) of the initial gauge-invariant Hamiltonian (2) was performed:

$$
-g \hat{p} \hat{s}_{i}^{z}=-i \sqrt{\frac{\hbar \omega}{2 V}}\left(\hat{a}^{\dagger}-\hat{a}\right) 2 e l \sqrt{4 \pi} \hat{s}_{i}^{z}=-\hat{\mathscr{E}} \hat{\vec{d}}_{i},
$$

which represents the energy of the dipole in the electric field. The electric-field operator in (11) is given by

$$
\hat{\mathscr{E}}=i \sqrt{\frac{h \omega}{V}}\left(\hat{a}^{\dagger}-\hat{a}\right) \vec{\epsilon}
$$

and the dipole moment of the single junction is

$$
\hat{\vec{d}}_{i}=2 e \hat{s}_{i}^{z} l \vec{\epsilon}
$$


The total dipole moment is then

$$
\hat{\vec{d}}=\sum_{i=1}^{N} \hat{\vec{d}}_{i}=2 e l \vec{\epsilon} \sum_{i=1}^{N} \hat{s}_{i}^{z}
$$

For convenience of further calculations we perform a unitary transformation $U^{\dagger} \hat{H} U$, where $U=\frac{1}{\sqrt{2}}\left(\begin{array}{cc}1 & i \\ 1 & -i\end{array}\right)$ interchanges operators of the Cartesian components of spin 1/2: $\hat{s}_{i}^{z} \rightarrow-\hat{s}_{i}^{y}, \hat{s}_{i}^{y} \rightarrow-\hat{s}_{i}^{x}$, and $\hat{s}_{i}^{x} \rightarrow \hat{s}_{i}^{z}$.

Hence, the final Hamiltonian of the Cooper pair boxes, that we are going to explore, becomes

$$
\hat{H}=\frac{1}{2}\left(\hat{p}^{2}+\omega^{2} \hat{q}^{2}\right)+g \hat{p} \hat{S}^{y}-E_{J} \hat{S}^{z}+\frac{g^{2}}{2}\left(\hat{S}^{y}\right)^{2},
$$

where we have introduced operators $\hat{S}^{\alpha}=\sum_{i} \hat{s}_{i}^{\alpha}$ of the total spin components. The total spin $\hat{S}^{2}$ is conserved, because it commutes with (15): $\left[\hat{S}^{2}, \hat{H}\right]=0$. Cooper pairs tunneling is represented by the $-E_{J} \hat{S}^{z}$ term, $g \hat{p} \hat{S}^{y}$ is a dipole coupling strength of a Cooper pair box to the photonic field, and $\left(g^{2} / 2\right)\left(\hat{S}^{y}\right)^{2}$ stands for the infinitely coordinated "antiferromagnetic" frustrating term.

\section{DIAGONALIZATION OF THE FRUSTRATED DICKE MODEL}

\section{A. Tunneling regime}

In this section, we consider the frustrated Dicke Hamiltonian (15) and first assume that at small coupling strength $g$ the Josephson tunneling term $-E_{J} \hat{S}_{z}$ dominates at zero temperature. Then the superspin is in the large $S$ sector and hence one is allowed to use the Holstein-Primakoff (HP) transformation [26] in the form

$$
\begin{aligned}
& \hat{S}^{z}=S-\hat{b}^{\dagger} \hat{b}, \\
& \hat{S}^{y}=i \sqrt{\frac{S}{2}}\left(\hat{b}^{\dagger} \sqrt{1-\frac{\hat{b}^{\dagger} \hat{b}}{2 S}}-\sqrt{1-\frac{\hat{b}^{\dagger} \hat{b}}{2 S}} \hat{b}\right) \simeq i \sqrt{\frac{S}{2}}\left(\hat{b}^{\dagger}-\hat{b}\right), \\
& \hat{S}^{x}=\sqrt{\frac{S}{2}}\left(\hat{b}^{\dagger} \sqrt{1-\frac{\hat{b}^{\dagger} \hat{b}}{2 S}}+\sqrt{1-\frac{\hat{b}^{\dagger} \hat{b}}{2 S}} \hat{b}\right) \simeq \sqrt{\frac{S}{2}}\left(\hat{b}^{\dagger}+\hat{b}\right),
\end{aligned}
$$

where $\left[\hat{b}, \hat{b}^{\dagger}\right]=1$. The substitution of (16) and (17) into (15) gives the Hamiltonian of the two linearly coupled harmonic oscillators:

$$
\begin{aligned}
\hat{H}= & \omega\left(\hat{a}^{\dagger} \hat{a}+\frac{1}{2}\right)-E_{J}\left(S-\hat{b}^{\dagger} \hat{b}\right) \\
& -\frac{g \sqrt{S \omega}}{2}\left(\hat{a}^{\dagger}-\hat{a}\right)\left(\hat{b}^{\dagger}-\hat{b}\right)-\frac{g^{2} S}{4}\left(\hat{b}^{\dagger}-\hat{b}\right)^{2} .
\end{aligned}
$$

This model also arises in the case of the ultrastrong light-matter coupling regime with polariton dots [27]. Here and in what follows we set $\hbar=1$. With the help of the usual linear Bogoliubov transformation of the creation/annihilation operators (see Appendix A) we obtain the diagonalized Hamiltonian

$$
\begin{aligned}
\hat{H}= & -E_{J}\left(S+\frac{1}{2}\right)+\frac{1}{2}\left(\varepsilon_{1}+\varepsilon_{2}\right) \\
& +\varepsilon_{1} \hat{c}_{1}^{\dagger} \hat{c}_{1}+\varepsilon_{2} \hat{c}_{2}^{\dagger} \hat{c}_{2}
\end{aligned}
$$

with the excitations spectrum described by the new oscillator frequencies

$$
\begin{aligned}
2 \varepsilon_{1,2}^{2}= & E_{J}\left(E_{J}+g^{2} S\right)+\omega^{2} \\
& \pm \sqrt{\left(E_{J}\left(E_{J}+g^{2} S\right)-\omega^{2}\right)^{2}+4 \omega^{2} g^{2} S E_{J}},
\end{aligned}
$$

where the frequencies $\varepsilon_{1,2}$ have to be chosen positive to keep the Hermiticity of the initial operators $\hat{p}, \hat{q}$, and $\hat{S}_{y}$. In contrast with the Dicke model without frustration [23] both energy branches are real in the whole range of the coupling constants $g$, but with a caveat. Namely, the ground-state energy equals

$$
E_{0}(S)=-E_{J}\left(S+\frac{1}{2}\right)+\frac{1}{2}\left(\varepsilon_{1}+\varepsilon_{2}\right) .
$$

This ground state is stable as long as the ground-state energy $E_{0}(S)$ (22) has a global minimum as a function of the superspin $S$ at the end of the interval [0,N/2]. One can find the value of the coupling strength $g=\tilde{g}$, at which the minimum becomes double degenerate, via solving equation $E_{0}(S=N / 2, g=\tilde{g})=E_{0}(S=0, g=\tilde{g})=\omega / 2$ :

$$
\tilde{g} \simeq \sqrt{2 E_{J} N}+\left(E_{J}+\omega\right) \sqrt{\frac{2}{N E_{J}}},
$$

which can be easily derived from large $g$ asymptotic expression of $E_{0}(S)$ :

$$
E_{0} \simeq-E_{J}\left(S+\frac{1}{2}\right)+\frac{g}{2} \sqrt{E_{J} S} .
$$

For $g>\tilde{g}$ the minimum of $E_{0}(S)$ migrates from $S=N / 2$ to 0 (see Fig. 1). This "jump" of the minimum obviously makes ground state $S=N / 2$ unstable and leads to an inapplicability of the quasiclassical HP approximation. Thus, our large $S$ ground-state description (22) is justified for $g<\tilde{g}$.

\section{B. Rotating Holstein-Primakoff representation}

In order to continue the theory into the strong-coupling regime, i.e., outside the interval $g<\tilde{g}$, we substitute in the

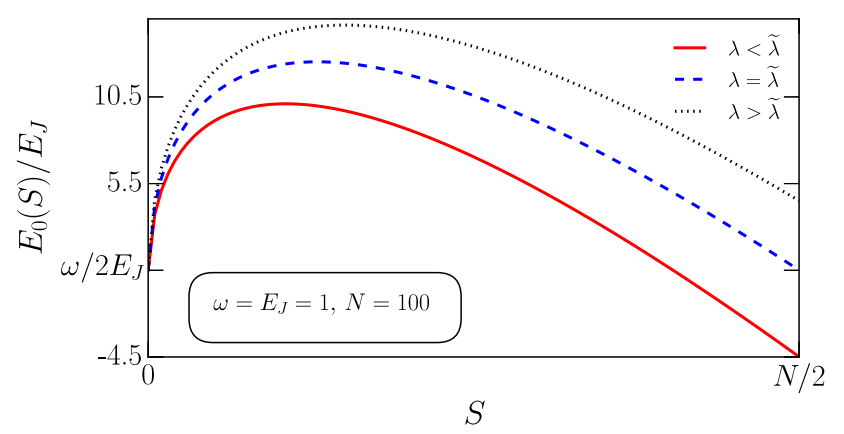

FIG. 1. Ground-state energy as a function of the superspin $S$ at fixed dimensionless coupling constant $\lambda=g \sqrt{N / 2 E_{J}}$. The blue dashed line shows the double degenerate minima of the ground state at the coupling strength $\lambda=\tilde{\lambda}$ (23). 
Hamiltonian (15) the $y$ and $z$ components of the total spin operators with a generalized expression of the Holstein-Primakoff representation in a coordinate frame rotated by an angle $\theta$ in the $z-y$ plane:

$$
\begin{aligned}
& \hat{S}^{z}=\hat{J}^{z} \cos \theta-\hat{J}^{y} \sin \theta, \\
& \hat{S}^{y}=\hat{J}^{z} \sin \theta+\hat{J}^{y} \cos \theta .
\end{aligned}
$$

Here the set of operators of the Cartesian projections of the total spin $\hat{J}^{x, y, z}$ is

$$
\begin{aligned}
& \hat{J}^{z}=S-\hat{b}^{\dagger} \hat{b}, \\
& \hat{J}^{y} \simeq i \sqrt{\frac{S}{2}\left(\hat{b}^{\dagger}-\hat{b}\right),} \\
& \hat{J}^{x} \simeq \sqrt{\frac{S}{2}}\left(\hat{b}^{\dagger}+\hat{b}\right) .
\end{aligned}
$$

To find the $\theta \neq 0$ solution that diagonalizes (15) we introduce a shift, $i \sqrt{\alpha}$, of the photon creation/annihilation operators, similar to [23], in the following way:

$$
\begin{aligned}
\hat{a}^{\dagger} & =\hat{c}^{\dagger}-i \sqrt{\alpha}, \\
\hat{a} & =\hat{c}+i \sqrt{\alpha},
\end{aligned}
$$

thus envisaging formation of a super-radiant state. After we substitute (25)-(27) into (15), the Hamiltonian, quadratic in operators $c, c^{\dagger}, b$, and $b^{\dagger}$, becomes

$$
\begin{aligned}
\hat{H}= & \omega\left(\hat{c}^{\dagger} \hat{c}+\frac{1}{2}\right)-E_{J} \cos \theta\left(S-\hat{b}^{\dagger} \hat{b}\right) \\
& -\frac{g \cos \theta \sqrt{S \omega}}{2}\left(\hat{c}^{\dagger}-\hat{c}\right)\left(\hat{b}^{\dagger}-\hat{b}\right)-\frac{g^{2} \cos ^{2} \theta S}{4}\left(\hat{b}^{\dagger}-\hat{b}\right)^{2},
\end{aligned}
$$

where an elimination of the linear in $\left(\hat{c}^{\dagger}-\hat{c}\right)$ and $\left(\hat{b}^{\dagger}-\hat{b}\right)$ terms in the Hamiltonian introduces a system of the two equations:

$$
\begin{gathered}
\sqrt{2 \omega \alpha}+g \sin \theta\left(S-\left\langle\hat{b}^{\dagger} \hat{b}\right\rangle\right)=0, \\
E_{J} \sin \theta+g \cos \theta \sqrt{2 \omega \alpha}+g^{2} \cos \theta \sin \theta\left(S-\left\langle\hat{b}^{\dagger} \hat{b}\right\rangle-\frac{1}{2}\right)=0 .
\end{gathered}
$$

We have also made in (28) a mean-field decoupling of the products that are higher than quadratic in $b$ and $b^{\dagger}$ operators: $\hat{b}^{\dagger} \hat{b} \hat{b}^{\dagger} \hat{b}=2\left\langle\hat{b}^{\dagger} \hat{b}\right\rangle \hat{b}^{\dagger} \hat{b}-\left\langle\hat{b}^{\dagger} \hat{b}\right\rangle^{2}$ and $\hat{b}^{\dagger} \hat{b}\left(\hat{b}^{\dagger}-\hat{b}\right)+$ $\left(\hat{b}^{\dagger}-\hat{b}\right) \hat{b}^{\dagger} \hat{b}=\left(\hat{b}^{\dagger}-\hat{b}\right)\left(1+2\left\langle\hat{b}^{\dagger} \hat{b}\right\rangle\right)$.

Nontrivial solutions $\alpha \neq 0$ and $\theta \neq 0$ of the system of equations (29) and (30) emerge when $g \geqslant \sqrt{2 E_{J}}$ :

$$
\begin{gathered}
\cos \theta=\frac{2 E_{J}}{g^{2}} \\
\sqrt{\alpha}=-\frac{g S}{\sqrt{2 \omega}}\left(1-\frac{\left\langle\hat{b}^{\dagger} \hat{b}\right\rangle}{S}\right) \sin \theta \simeq-\frac{g S}{\sqrt{2 \omega}} \sin \theta \\
=\frac{g S}{\sqrt{2 \omega}} \sqrt{1-\frac{4 E_{J}^{2}}{g^{4}}}
\end{gathered}
$$

and

$$
\sqrt{\alpha} \rightarrow-\sqrt{\alpha} ; \theta \rightarrow-\theta .
$$

Under the solutions (31)-(33), the energy of the photonic condensate $=\omega \alpha$ exactly cancels with the sum of the rest of the $c$-number terms in the Hamiltonian (28):

$$
\begin{aligned}
\omega \alpha & +g S \sin \theta \sqrt{2 \omega \alpha}+\frac{g^{2} \sin ^{2} \theta}{2}\left(S^{2}-\left\langle\hat{b}^{\dagger} \hat{b}\right\rangle^{2}\right) \\
= & \frac{g^{2} \sin ^{2} \theta}{2}\left(S^{2}-2 S\left\langle\hat{b}^{\dagger} \hat{b}\right\rangle+\left\langle\hat{b}^{\dagger} \hat{b}\right\rangle^{2}-2 S^{2}\right. \\
& \left.+2 S\left\langle\hat{b}^{\dagger} \hat{b}\right\rangle+S^{2}-\left\langle\hat{b}^{\dagger} \hat{b}\right\rangle^{2}\right)=0 .
\end{aligned}
$$

The $c$-number terms in the first line of (34) have the following meaning: the photonic condensate energy $\sim \omega \alpha$, the (negative) contribution of the dipole-photon coupling energy $\sim g\langle\hat{p}\rangle\left\langle\hat{S}^{y}\right\rangle$, and the zero-point oscillations energy of the frustrating term $\sim g^{2}\left\langle\left(\hat{S}^{y}\right)^{2}\right\rangle / 2$. The total of these three terms proves to be zero. This $\alpha$-independent cancellation, actually, stems from the degeneracy of the energy minima of the diagonal in the spin operators part of the extended Dicke Hamiltonian (15) with respect to $2 S+1$ different $\hat{S}^{y}$ projections and classical part $\sim \sqrt{\alpha}$ of the photonic operator $\hat{p}$.

\section{Super-radiant dipolar regime}

The structure of (28) is the same as (19), though with coefficients renormalized with prefactor $\cos \theta$ due to RHP rotation by an angle $\theta$. Hence, after a Bogoliubov transformation similar to the one already described in the Appendix A, the diagonalized Hamiltonian expressed via new second quantized operators $\hat{e}_{1,2}^{\dagger}$ and $\hat{e}_{1,2}$ acquires the form

$$
\begin{aligned}
\hat{H}= & -\frac{g^{2} \cos ^{2} \theta}{2}\left(S+\frac{1}{2}\right)+\frac{1}{2}\left(\tilde{\varepsilon}_{1}+\tilde{\varepsilon}_{2}\right) \\
& +\tilde{\varepsilon}_{1} \hat{e}_{1}^{\dagger} \hat{e}_{1}+\tilde{\varepsilon}_{2} \hat{e}_{2}^{\dagger} \hat{e}_{2}
\end{aligned}
$$

with the positive eigenvalues $\tilde{\varepsilon}_{1,2}$,

$$
\begin{aligned}
2 \tilde{\varepsilon}_{1,2}^{2}= & \frac{g^{4} \cos ^{4} \theta}{2}\left(S+\frac{1}{2}\right)+\omega^{2} \\
& \pm \sqrt{\left[\frac{g^{4} \cos ^{4} \theta}{2}\left(S+\frac{1}{2}\right)-\omega^{2}\right]^{2}+2 S \omega^{2} g^{4} \cos ^{4} \theta}
\end{aligned}
$$

and the ground-state energy

$$
\widetilde{E}_{0}(S)=-\frac{g^{2} \cos ^{2} \theta}{2}\left(S+\frac{1}{2}\right)+\frac{1}{2}\left(\tilde{\varepsilon}_{1}+\tilde{\varepsilon}_{2}\right) .
$$

The stability of the large $S$ state in this regime is provided by the negative slope of $E_{0}(S)$ as a function of $S$ (see Fig. 2) in the strong-coupling limit:

$$
\left.\widetilde{E}_{0}(S)\right|_{g \rightarrow+\infty} \simeq \frac{\omega}{2}-\frac{2 E_{J}^{2}}{g^{2}}\left(S+\frac{1}{2}\right) .
$$

The interval $g \geqslant \sqrt{2 E_{J}}$ is characterized with an emergent dipole moment of the Cooper pair boxes array and the superradiant photonic condensate either as a metastable state for $\sqrt{2 E_{J}} \leqslant g<g_{c}$ or as the ground state for $g \geqslant g_{c}$ (the critical 


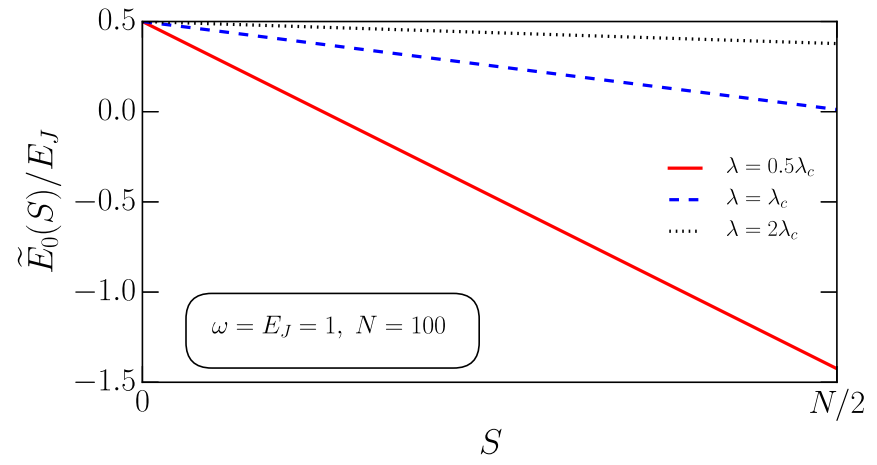

FIG. 2. Ground-state energy $\widetilde{E}_{0}$ as a function of the superspin $S$ at fixed dimensionless coupling constant $\lambda=g \sqrt{N / 2 E_{J}}$.

strength $g_{c}$ is found below). To see this explicitly, we express electromagnetic field operators via a new set of Bose operators found after the Bogoliubov transformation:

$$
\begin{gathered}
\hat{p}=\sqrt{2 \omega \alpha}+i \frac{\omega \cos \delta}{\sqrt{2 \tilde{\varepsilon}_{1}}}\left(\hat{e}_{1}^{\dagger}-\hat{e}_{1}\right)+i \frac{\omega \sin \delta}{\sqrt{2 \tilde{\varepsilon}_{2}}}\left(\hat{e}_{2}^{\dagger}-\hat{e}_{2}\right) \\
\hat{q}=\frac{\cos \delta}{\sqrt{2 \tilde{\varepsilon}_{1}}}\left(\hat{e}_{1}^{\dagger}+\hat{e}_{1}\right)+\frac{\sin \delta}{\sqrt{2 \tilde{\varepsilon}_{2}}}\left(\hat{e}_{2}^{\dagger}+\hat{e}_{2}\right)
\end{gathered}
$$

In turn, the spin operators are expressed via $\hat{e}_{i}, \hat{e}_{i}^{\dagger}, i=1,2$ as well:

$$
\begin{gathered}
\hat{J}_{z}=S\left(1-\frac{\left\langle\hat{b}^{\dagger} \hat{b}\right\rangle}{S}\right) \simeq S \\
\hat{J}_{y}=-i \frac{E_{J} \sqrt{S} \sin \delta}{g \sqrt{\tilde{\varepsilon}_{1}}}\left(\hat{e}_{1}^{\dagger}-\hat{e}_{1}\right)+i \frac{E_{J} \sqrt{S} \cos \delta}{g \sqrt{\tilde{\varepsilon}_{2}}}\left(\hat{e}_{2}^{\dagger}-\hat{e}_{2}\right) \\
\hat{J}_{x}=-\frac{E_{J} \sqrt{S} \sin \delta}{g \sqrt{\tilde{\varepsilon}_{1}}}\left(\hat{e}_{1}^{\dagger}+\hat{e}_{1}\right)+\frac{E_{J} \sqrt{S} \cos \delta}{g \sqrt{\tilde{\varepsilon}_{2}}}\left(\hat{e}_{2}^{\dagger}+\hat{e}_{2}\right)
\end{gathered}
$$

The Bogoliubov "angle" $\delta$ can be found from the consistency relation

$$
\tan 2 \delta=\frac{2 \sqrt{2 S} \omega g^{2} \cos ^{2} \theta}{g^{4} \cos ^{4} \theta\left(S+\frac{1}{2}\right)-2 \omega^{2}} .
$$

We find for $g \geqslant \sqrt{2 E_{J}}$ the following nonzero expectation values in the ground state of Hamiltonian (35). For the electric field $\hat{\vec{E}}$,

$$
\begin{aligned}
\langle\hat{\mathscr{E}} \cdot \vec{\epsilon}\rangle \sqrt{\frac{V}{4 \pi}} & =\langle\hat{p}\rangle=\sqrt{2 \omega \alpha} \simeq-g S \sin \theta \\
& =\mp g S \sqrt{1-\frac{4 E_{J}^{2}}{g^{4}}}
\end{aligned}
$$

for the modulus of the Josephson tunneling energy of the Cooper pairs (it decreases),

$$
-E_{J}\left\langle\hat{S}^{z}\right\rangle=-E_{J}\left\langle\hat{J}^{z}\right\rangle \cos \theta \simeq-S \frac{2 E_{J}^{2}}{g^{2}} ;
$$

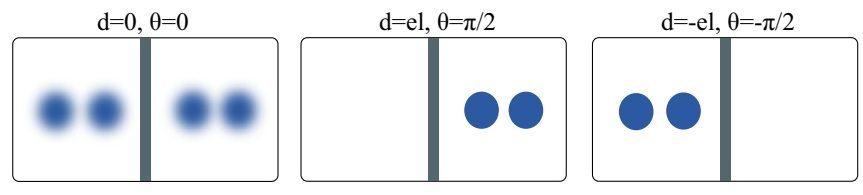

FIG. 3. Schematic layout of the amplitude distributions of the Cooper pair's wave function in the adjacent islands of a single $\mathrm{JJ}$ and corresponding dipole moment values depending on the rotation angle $\theta$ [see text and Eqs. (25)].

and, for the emergent finite mean value of the dipole moment,

$$
\langle\hat{d}\rangle=2 e l\left\langle\hat{S}^{y}\right\rangle=2 e l\left\langle\hat{J}^{z}\right\rangle \sin \theta \simeq \pm 2 e l S \sqrt{1-\frac{4 E_{J}^{2}}{g^{4}}} .
$$

Hence, results (45) and (47) indicate that upon an increase of the coupling strength $g>\sqrt{2 E_{J}}$ there is a state with the energy given in (37), which is characterized by an emergent super-radiant electromagnetic field $\langle\hat{p}\rangle \neq 0$ in the cavity together with a finite dipole moment of the Cooper pair boxes: $\langle\hat{d}\rangle \neq 0$. The latter means that rotation angle $\theta$ introduced in (25) regulates an extent of a Cooper pair wave function between the superconducting islands forming each Josephson junction in the Josephson-junction array (see Fig. 3). Namely, when $\theta$ progressively deviates from zero, the Cooper pairs become localized in one of the two superconducting islands constituting a given Josephson junction, and as a result the latter acquires a dipole moment.

\section{FIRST-ORDER DIPOLAR PHASE TRANSITION}

In this section we calculate a critical coupling $g_{c}$, at which a first-order phase transition between the tunneling and dipolar states described in Secs. III A and III C takes place.

In Fig. 4 we plotted ground-state energies calculated for tunneling and dipolar states as functions of coupling $g: E_{0}(S)$ and $\widetilde{E}_{0}(S)$ [see (22) and (37) correspondingly]. A dimensionless coupling constant $\lambda=g \sqrt{N / 2 E_{J}}$ is used. In the strong-coupling limit, $g \gg \sqrt{2 E_{J}}$, the $g$ dependence of both branches of energy is very well approximated by (24) and (38).

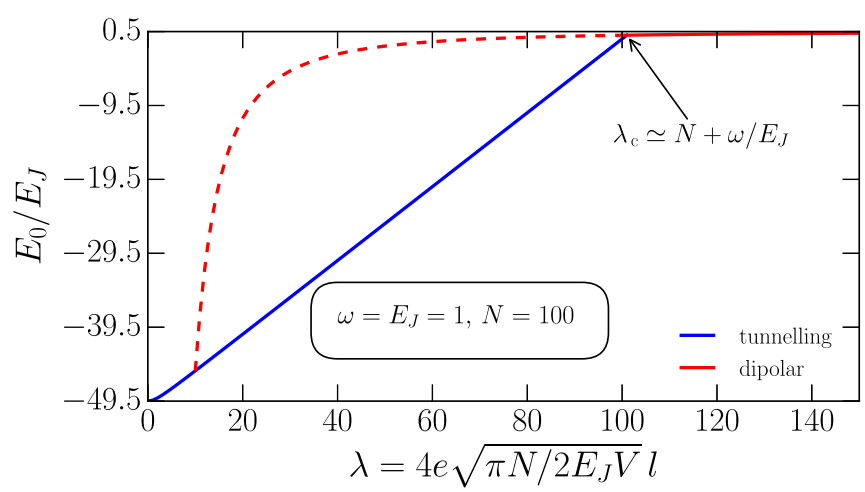

FIG. 4. Ground-state energy as a function of dimensionless coupling constant $\lambda=g \sqrt{N / 2 E_{J}}$. The blue line is for the Josephson tunneling state in the interval $\lambda<\lambda_{\mathrm{c}} \approx N$. The red line is for the dipolar ordered state. The red dashed line shows the dipolar state in the metastable region preceding the first-order phase transition at $\lambda_{\mathrm{c}}$. 


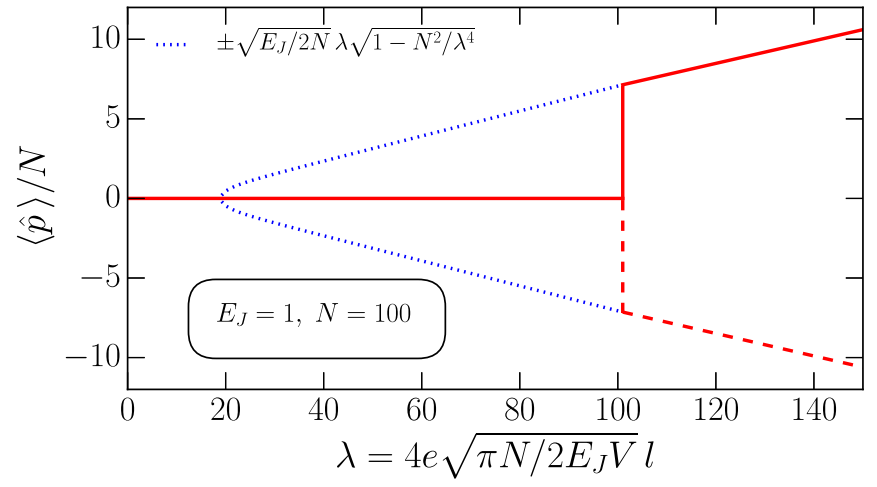

FIG. 5. Photon field $\langle\hat{p}\rangle$ emerging in the cavity as a function of dimensionless coupling constant $\lambda=g \sqrt{N / 2 E_{J}}$. The first-order transition to a state with macroscopic photon occupation number $\left\langle\hat{a}^{\dagger} \hat{a}\right\rangle \neq 0$ occurs at a critical coupling $\lambda_{\mathrm{c}} \simeq N+\omega / E_{J}$. The blue dotted line shows a metastable solution for $\langle\hat{p}\rangle$, that first appears at $\lambda=\sqrt{N}$.

Hence, in the thermodynamic limit $N \rightarrow \infty$, the solution of $E_{0}=\widetilde{E}_{0}$ gives the critical value $g_{\mathrm{c}}$ of the coupling constant:

$$
g_{\mathrm{c}} \simeq \sqrt{2 E_{J} N}+\omega \sqrt{\frac{2}{N E_{J}}} .
$$

Here a crucial difference with respect to [23] is that the critical point corresponds to $\lambda_{\mathrm{c}} \approx N$ and not 1 as in the standard Dicke model without frustration. Hence, transition now is size dependent, where the "size" of the system is the total number $N$ of Cooper pair boxes inside the microwave cavity.

At $\lambda=\sqrt{N}$, i.e., $g=\sqrt{2 E_{J}}$, the ground state becomes degenerate and a dipolar branch $\widetilde{E}_{0}(S)$ first appears. For $\sqrt{N}<\lambda<N$, i.e., $\sqrt{2 E_{J}}<g<g_{c}$, the dipolar state minimal energy $\widetilde{E}_{0}(S)$ is higher than the tunneling ground-state energy $E_{0}(S)$. Hence, the system remains in the tunneling state (i.e., dipolar disordered). At $\lambda=\lambda_{\mathrm{c}}$ the ground-state energy $E_{0}(S)$ crosses the dipole state energy branch $\widetilde{E}_{0}(S)$ for the second time and goes above $\widetilde{E}_{0}(S)$. At the critical coupling $g=g_{\text {c }}$ (i.e., $\lambda=\lambda_{\mathrm{c}}$ ) the first-order phase transition from the tunneling state to dipolar ordered state takes place. It is, indeed, a first-order transition, since at $g=g_{\mathrm{c}}$ the dipole moment in the dipolar state is already finite: $\langle\hat{d}\rangle \approx \pm 2 e l S$ [see (47)], while in the tunneling state it equals zero. Namely, the first-order phase transition results in

$$
\langle\hat{p}\rangle=-S g \sin \theta=\left\{\begin{array}{l}
0, \quad g<g_{\mathrm{c}} \\
\mp S g \sqrt{1-4 E_{J}^{2} / g^{4}}, \quad g \geqslant g_{\mathrm{c}}
\end{array}\right.
$$

(see Fig. 5) and

$$
\begin{aligned}
& -E_{J}\left\langle\hat{S}^{z}\right\rangle=-S E_{J} \cos \theta=\left\{\begin{array}{l}
-S E_{J}, \quad g<g_{\mathrm{c}} \\
-E_{J}^{2} 2 S / g^{2}, \quad g \geqslant g_{\mathrm{c}}
\end{array},\right. \\
& \langle\hat{d}\rangle=2 e l S \sin \theta=\left\{\begin{array}{l}
0, \quad g<g_{\mathrm{c}} \\
\pm 2 e l S \sqrt{1-4 E_{J}^{2} / g^{4}}, \quad g \geqslant g_{\mathrm{c}}
\end{array}\right.
\end{aligned}
$$

The collective dipole moment (51) is defined by the angle $\theta$, which is shown in Fig. 6.

It is important to mention here that comparison of (48) with (23) gives $\tilde{g}-g_{\mathrm{c}}=\sqrt{2 E_{J} / N}>0$. Hence, we have

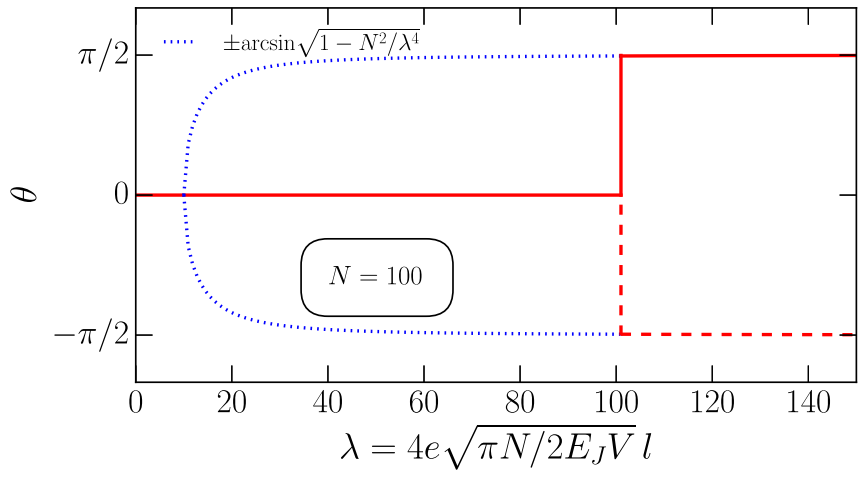

FIG. 6. The angle $\theta$, that characterizes rotation of HP, as a function of dimensionless coupling constant $\lambda=g \sqrt{N / 2 E_{J}}$. The color scheme is chosen the same as for Fig. 5.

found the first-order phase transition in the region of validity (i.e., $g<\tilde{g}$ ) of the large superspin limit $S=N / 2 \gg 1$, that justifies the use of the HP approach. In the limit $g \rightarrow+\infty$ the dipolar ordered ground-state energy $\widetilde{E}_{0}$ approaches from below the ground-state energy of a free resonant photon, $\omega / 2$. Simultaneously, at $g=g_{\text {c }}$ the ground-state energy $\widetilde{E}_{0}(S)=$ $0<\omega / 2$. Hence, our semiclassical description indicates that after the dipole transition the system gradually approaches the "decoupled state" $\widetilde{E}_{0}(S)=\omega / 2$, but with saturated values of the collective dipole moment $\propto\left\langle\hat{S}^{y}\right\rangle \rightarrow N / 2$ and photon occupation number $\alpha \propto\langle\hat{p}\rangle^{2} \rightarrow N^{2} g^{2} / \omega$. It is not possible to decide in the framework of our semiclassical approach whether a crossover to a state $\left\langle\hat{S}^{y}\right\rangle=0$ happens in the $g \rightarrow+\infty$ limit. The latter state was predicted numerically in finite or even $N$ spin-1/2 cluster realization of the extended Dicke model [6].

The excitation branches (36) of the diagonalized Hamiltonian are shown in Figs. 7 and 8.

The branch $\varepsilon_{1}$, that grows with the increase of the coupling, goes to the initial photon's frequency $\omega$ after the first-order transition. The branch $\varepsilon_{2}$ approaches zero in the strongcoupling limit.

Combining together (48) and expression $g=$ $2 e l \sqrt{4 \pi} /(\sqrt{V})$, one may formulate a condition for an

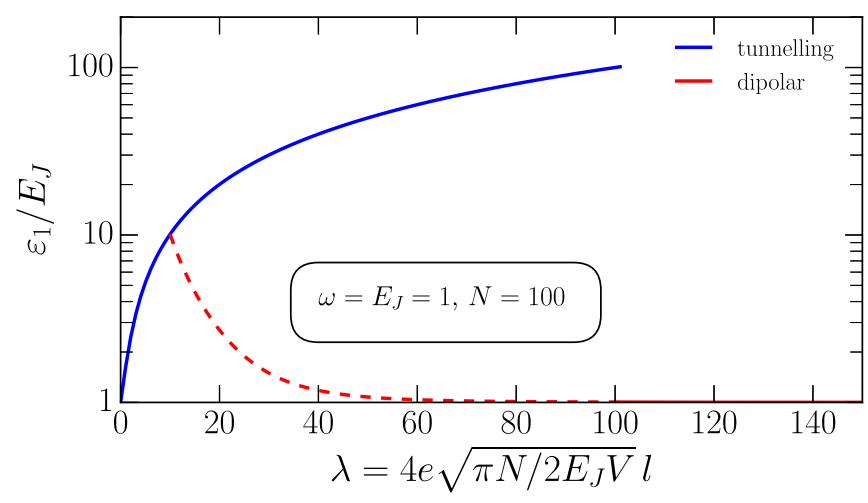

FIG. 7. Excitation branches $\varepsilon_{1}$ and $\tilde{\varepsilon}_{1}$ [Eqs. (21) and (36)] as the functions of dimensionless coupling constant $\lambda=g \sqrt{N / 2 E_{J}}$. The vertical axis is shown in the logarithmic scale. At the critical coupling $\lambda_{\mathrm{c}} \approx N$ the frequency $\varepsilon_{1}$ falls down to $\tilde{\varepsilon}_{1} \approx \omega$. The color scheme is the same as in Fig. 4. 


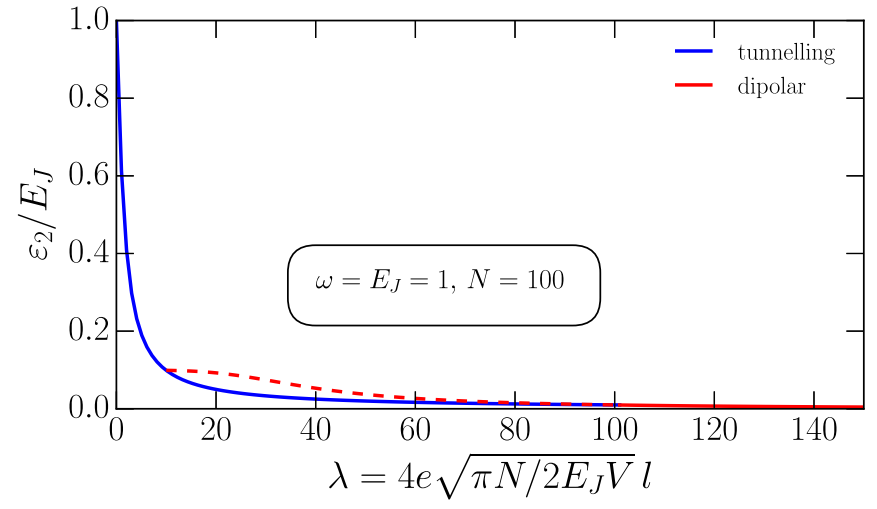

FIG. 8. Excitation branches $\varepsilon_{2}$ and $\tilde{\varepsilon}_{2}$ [Eqs. (21) and (36)] as a function of dimensionless coupling constant $\lambda=g \sqrt{N / 2 E_{J}}$. The frequencies $\varepsilon_{2}$ and $\tilde{\varepsilon}_{2}$ asymptotically approach zero in the strongcoupling limit. The color scheme is the same as in Fig. 4.

occurrence of the dipolar quantum phase transition:

$$
4 e l \sqrt{\pi / V}=\sqrt{2 N E_{J}}
$$

where $l$ is a penetration depth of electric field into the Cooper pair box superconducting island, and $V$ is the volume of the microwave cavity. Taking into account that charging energy $E_{C}=(2 e)^{2} / 2 C$ is of order $E_{C}=2 e^{2} / l$, one may rewrite (52) in the following form:

$$
L \approx l \frac{E_{C} l^{2}}{E_{J} N \Sigma},
$$

where $\Sigma$ and $L$ are waveguide (microwave cavity) crosssection area and length, respectively. Assuming $L \approx N l$ we finally find the following condition:

$$
N^{2} \approx \frac{E_{C} l^{2}}{E_{J} \Sigma} .
$$

Hence, we come to a similar conclusion (see Fig. 9) as was already made in [7], that in order to achieve strong-coupling limit $g \geqslant g_{\text {c }}$ for a Cooper pair boxes array of a "thermodynamic size" $N \approx 10^{2}$ inside a microwave resonator a coplanar geometry with one-dimensional superconducting transmission line

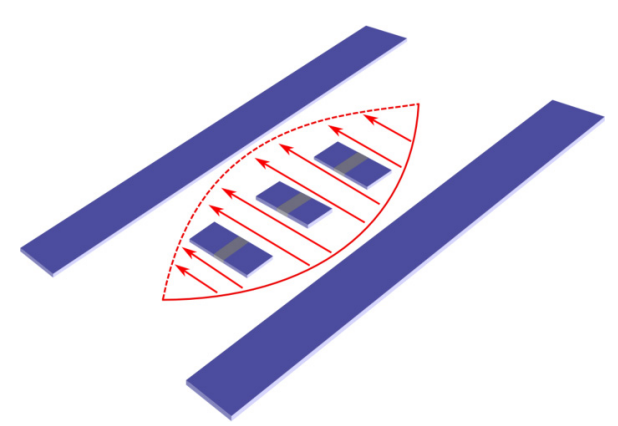

FIG. 9. Schematic layout of a Cooper pair boxes array inside a microwave resonator of coplanar geometry with one-dimensional superconducting transmission line (stripline resonator), similar to that proposed in [7] for achieving of a strong coupling $g$ between two-level systems and resonant photons in a model with the frustrated Dicke Hamiltonian.

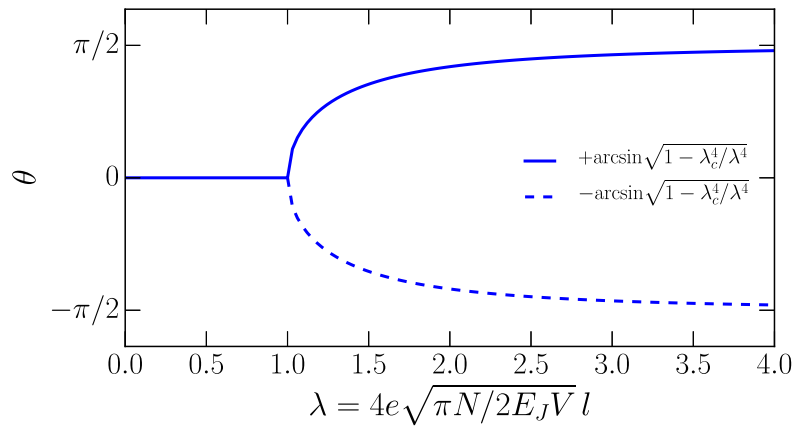

FIG. 10. The angle $\theta$ as a function of dimensionless coupling constant $\lambda=g \sqrt{N / 2 E_{J}}$ in the Dicke model without the frustrating interaction term.

(stripline resonator) should be used, thus providing inequality $\Sigma / l^{2} \ll 1$, and the Cooper pair box should have charging energy much greater than Josephson coupling energy: $E_{C} \gg$ $E_{J}$.

\section{CONCLUSIONS}

In summary, we have demonstrated that strong enough capacitive coupling of the Cooper pair boxes array of lowcapacitance Josephson junctions to microwave resonant photons may lead to a first-order quantum phase transition. As a result, a dipolar ordered state of Cooper pairs is formed, coupled to the emerged coherent photonic condensate. The phase transition is of the first order due to infinitely coordinated antiferromagnetic (frustrating) interaction, that arises between Cooper pair dipoles of different Cooper pair boxes. This frustrating interaction is induced by a gauge-invariant coupling of the Josephson junctions to a vector potential of the resonant photons in the microwave cavity. The strength of the coherent electromagnetic radiation field that emerges under the phase transition is proportional to the number $N$ of the Cooper pair boxes in the array and is reminiscent of the super-radiant state of the Dicke model without frustrating term found previously [23]. Nevertheless, the phase transition into the latter state is of second order [23] (see also Fig. 10 and Appendix B).

The analytical description of the first-order quantum phase transition in the Dicke model with infinitely coordinated antiferromagnetic frustrating interaction is made possible by an analytic tool: self-consistently "rotating" Holstein-Primakoff representation for the Cartesian components of the total spin, which is described in this paper. Our approach enables, as a byproduct, a description of the second-order quantum phase transition in the Dicke model without frustrating antiferromagnetic interaction, explored previously by other authors [23]. Nevertheless, rotating Holstein-Primakoff representation remains semiclassical $(S \rightarrow \infty)$. Therefore, the region of "spin liquid" with $S \sim 1$ is not attainable within this method.

\section{ACKNOWLEDGMENTS}

The authors acknowledge illuminating discussions with Carlo Beenakker, Konstantin Efetov, and Bernard van Heck during the course of this work. This research was supported 
by the Netherlands Organization for Scientific Research, a European Research Council Synergy grant, the Russian Ministry of Education and Science via the Increase Competitiveness Program of National University of Science and Technology "MISIS," Grant No. K2-2017-085, and "Goszadaniye" Grant No. 3.3360.2017/PH.

\section{APPENDIX A: BOGOLIUBOV'S TRANSFORMATION FOR THE FRUSTRATED HAMILTONIAN}

Below we show in detail a diagonalization procedure of the Hamiltonian (19). Let us introduce

$$
\hat{p}_{x}=i \frac{1}{\sqrt{2 \omega}}\left(\hat{a}^{\dagger}-\hat{a}\right) \text { and } \hat{x}=\sqrt{\frac{\omega}{2}}\left(\hat{a}^{\dagger}+\hat{a}\right)
$$

together with

$$
\hat{p}_{y}=i \frac{1}{\sqrt{2 E_{J}}}\left(\hat{b}^{\dagger}-\hat{b}\right) \text { and } \hat{y}=\sqrt{\frac{E_{J}}{2}}\left(\hat{b}^{\dagger}+\hat{b}\right)
$$

and rewrite (19) in terms of (A1) and (A2):

$$
\begin{aligned}
\hat{H}= & -E_{J}\left(S+\frac{1}{2}\right)+\frac{1}{2}\left(\hat{x}^{2}+\omega^{2} \hat{p}_{x}^{2}\right)+\frac{1}{2}\left(\hat{y}^{2}+E_{J}^{2} \hat{p}_{y}^{2}\right) \\
& +\omega g \sqrt{S E_{J}} \hat{p}_{x} \hat{p}_{y}+\frac{g^{2} S E_{J}}{2} \hat{p}_{y}^{2} \\
= & -E_{J}\left(S+\frac{1}{2}\right)+\frac{1}{2} \hat{K}_{x y}+\frac{1}{2} \hat{K}_{p_{x} p_{y}}
\end{aligned}
$$

where

$$
\begin{gathered}
\hat{K}_{x y}=\hat{x}^{2}+\hat{y}^{2} \\
\hat{K}_{p_{x} p_{y}}=\omega^{2} \hat{p}_{x}^{2}+E_{J}\left(E_{J}+g^{2} S\right) \hat{p}_{y}^{2}+2 \omega g \sqrt{S E_{J}} \hat{p}_{x} \hat{p}_{y} .
\end{gathered}
$$

We diagonalize (A3) by performing a linear transformation of the quantum operators:

$$
\begin{aligned}
\left(\begin{array}{l}
\hat{p}_{x} \\
\hat{p}_{y}
\end{array}\right) & =\left(\begin{array}{cc}
\cos \gamma & \sin \gamma \\
-\sin \gamma & \cos \gamma
\end{array}\right)\left(\begin{array}{l}
\hat{p}_{1} \\
\hat{p}_{2}
\end{array}\right) \text { and } \\
\left(\begin{array}{l}
\hat{x} \\
\hat{y}
\end{array}\right) & =\left(\begin{array}{cc}
\cos \gamma & \sin \gamma \\
-\sin \gamma & \cos \gamma
\end{array}\right)\left(\begin{array}{l}
\hat{q}_{1} \\
\hat{q}_{2}
\end{array}\right) .
\end{aligned}
$$

Then

$$
\hat{K}_{x y}=\hat{q}_{1}^{2}+\hat{q}_{2}^{2}
$$

and

$$
\begin{aligned}
\hat{K}_{p_{x} p_{y}}= & {\left[\omega^{2} \cos ^{2} \gamma+E_{J}\left(E_{J}+g^{2} S\right) \sin ^{2} \gamma\right.} \\
& \left.-2 \omega g \sqrt{S E_{J}} \sin \gamma \cos \gamma\right] \hat{p}_{1}^{2} \\
& +\left[\omega^{2} \sin ^{2} \gamma+E_{J}\left(E_{J}+g^{2} S\right) \cos ^{2} \gamma\right. \\
& \left.+2 \omega g \sqrt{S E_{J}} \sin \gamma \cos \gamma\right] \hat{p}_{2}^{2} \\
& +\left\{\left[\omega{ }^{2}-E_{J}\left(E_{J}+g^{2} S\right)\right] \sin 2 \gamma\right. \\
& \left.+2 \omega g \sqrt{S E_{J}} \cos 2 \gamma\right\} \hat{p}_{1} \hat{p}_{2} .
\end{aligned}
$$

The diagonalization condition that eliminates the cross-term $\sim \hat{p}_{1} \hat{p}_{2}$ is

$$
\tan 2 \gamma=\frac{2 \omega g \sqrt{S E_{J}}}{E_{J}\left(E_{J}+g^{2} S\right)-\omega^{2}} .
$$

So, diagonalized operator $\hat{K}_{p_{x} p_{y}}$ becomes

$$
\hat{K}_{p_{x} p_{y}}=\varepsilon_{1}^{2} \hat{p}_{1}^{2}+\varepsilon_{2}^{2} \hat{p}_{2}^{2},
$$

where

$$
\begin{aligned}
2 \varepsilon_{1}^{2}= & E_{J}\left(E_{J}+g^{2} S\right)+\omega^{2}-\left[E_{J}\left(E_{J}+g^{2} S\right)-\omega^{2}\right] \cos 2 \gamma \\
& -2 \omega g \sqrt{S E_{J}} \sin 2 \gamma, \\
2 \varepsilon_{2}^{2}= & E_{J}\left(E_{J}+g^{2} S\right)+\omega^{2}+\left[E_{J}\left(E_{J}+g^{2} S\right)-\omega^{2}\right] \cos 2 \gamma \\
& +2 \omega g \sqrt{S E_{J}} \sin 2 \gamma .
\end{aligned}
$$

Substitution of (A9) into (A11) and (A12) gives the eigenvalues

$$
\begin{aligned}
2 \varepsilon_{1,2}^{2}= & E_{J}\left(E_{J}+g^{2} S\right)+\omega^{2} \\
& \pm \sqrt{\left[E_{J}\left(E_{J}+g^{2} S\right)-\omega^{2}\right]^{2}+4 \omega^{2} g^{2} S E_{J}}
\end{aligned}
$$

The transformation

$$
\hat{p}_{1,2}=i \frac{1}{\sqrt{2 \varepsilon_{1,2}}}\left(\hat{c}_{1,2}^{\dagger}-\hat{c}_{1,2}\right) \text { and } \hat{q}_{1,2}=\sqrt{\frac{\varepsilon_{1,2}}{2}}\left(\hat{c}_{1,2}^{\dagger}+\hat{c}_{1,2}\right)
$$

finally gives the diagonal Hamiltonian (20).

The initial operators $\hat{a}$ and $\hat{b}$ are expressed via the new operators $\hat{c}_{1,2}^{\dagger}$ as

and

$$
a=\sqrt{\frac{\omega}{\varepsilon_{1}}} \cos \gamma \hat{c}_{1}+\sqrt{\frac{\omega}{\varepsilon_{2}}} \sin \gamma \hat{c}_{2}
$$

$$
\hat{b}=-\sqrt{\frac{E_{J}}{\varepsilon_{1}}} \sin \gamma \hat{c}_{1}+\sqrt{\frac{E_{J}}{\varepsilon_{2}}} \cos \gamma \hat{c}_{2},
$$

where $\gamma$ is defined in (A9).

\section{APPENDIX B: QUANTUM PHASE TRANSITION OF SECOND ORDER IN THE DICKE MODEL WITHIN THE RHP METHOD}

We consider the standard Dicke Hamiltonian $[11,23]$ (modulo our notations)

$$
\hat{H}=\frac{1}{2}\left(\hat{p}^{2}+\omega^{2} \hat{q}^{2}\right)+g \hat{p} \hat{S}^{y}-E_{J} \hat{S}^{z}
$$

at small coupling $g$. We apply (16) and (17) to (B1):

$$
\begin{aligned}
\hat{H}= & -E_{J}\left(S+\frac{1}{2}\right)+\omega\left(\hat{a}^{\dagger} \hat{a}+\frac{1}{2}\right)+E_{J}\left(\hat{b}^{\dagger} \hat{b}+\frac{1}{2}\right) \\
& -\frac{g \sqrt{S \omega}}{2}\left(\hat{a}^{\dagger}-\hat{a}\right)\left(\hat{b}^{\dagger}-\hat{b}\right) .
\end{aligned}
$$

The Bogoliubov transformation, similar to those in Appendix A, gives

$$
\hat{H}=-E_{J}\left(S+\frac{1}{2}\right)+\varepsilon_{1}\left(\frac{1}{2}+\hat{c}_{1}^{\dagger} \hat{c}_{1}\right)+\varepsilon_{2}\left(\frac{1}{2}+\hat{c}_{2}^{\dagger} \hat{c}_{2}\right),
$$


with the excitations spectrum described by the new oscillator frequencies:

$$
2 \varepsilon_{1,2}^{2}=E_{J}^{2}+\omega^{2} \pm \sqrt{\left(E_{J}^{2}-\omega^{2}\right)^{2}+4 \omega^{2} g^{2} S E_{J}} .
$$

The ground-state energy equals

$$
E_{0}(S)=-E_{J}\left(S+\frac{1}{2}\right)+\frac{1}{2}\left(\varepsilon_{1}+\varepsilon_{2}\right)
$$

One can check that as a function of $S$ the energy $E_{0}(S)$ has a minimum at $S=N / 2$, i.e., at the end of the interval of all possible total spin values $0 \leqslant S \leqslant N / 2$. This fact justifies the Holstein-Primakoff approach (16)-(18) valid in the large spin limit.

However, the lowest branch of excitations becomes imaginary when $g>g_{\mathrm{c}}=\sqrt{E_{J} / S}$ :

$$
\varepsilon_{2}=\sqrt{\frac{E_{J}^{2}+\omega^{2}}{2}-\frac{\sqrt{\left(E_{J}^{2}-\omega^{2}\right)^{2}+4 \omega^{2} g^{2} S E_{J}}}{2}} .
$$

Thus, the ground state described above is unstable in the interval $g>g_{c}$ (compare [23]). The method described in Sec. III B [(25)-(27)] transforms the Hamiltonian (B2) into

$$
\begin{aligned}
\hat{H}= & \omega\left[\hat{c}^{\dagger} \hat{c}+i \sqrt{\alpha}\left(\hat{c}^{\dagger}-\hat{c}\right)+\alpha+\frac{1}{2}\right]-E_{J} \cos \theta\left(S-\hat{b}^{\dagger} \hat{b}\right) \\
& +E_{J} \sin \theta i \sqrt{\frac{S}{2}}\left(\hat{b}^{\dagger}-\hat{b}\right)-\frac{g \cos \theta \sqrt{S \omega}}{2}\left(\hat{c}^{\dagger}-\hat{c}\right)\left(\hat{b}^{\dagger}-\hat{b}\right) \\
& +g \cos \theta \sqrt{2 \omega \alpha} i \sqrt{\frac{S}{2}}\left(\hat{b}^{\dagger}-\hat{b}\right)+g \sin \theta i \sqrt{\frac{\omega}{2}}\left(\hat{c}^{\dagger}-\hat{c}\right) \\
& \times\left(S-\left\langle\hat{b}^{\dagger} \hat{b}\right\rangle\right)+g \sin \theta \sqrt{2 \omega \alpha}\left(S-\hat{b}^{\dagger} \hat{b}\right) .
\end{aligned}
$$

Here we have decoupled cubic in $\hat{c}$ and $\hat{b}$ operators terms in a mean-field approximation. Conditions for vanishing of the linear terms $\propto\left(\hat{c}^{\dagger}-\hat{c}\right)$ and $\left(\hat{b}^{\dagger}-\hat{b}\right)$ in the Hamiltonian (B7) are

$$
\begin{gathered}
\sqrt{2 \omega \alpha}+g \sin \theta\left(S-\left\langle\hat{b}^{\dagger} \hat{b}\right\rangle\right)=0, \\
E_{J} \sin \theta+g \cos \theta \sqrt{2 \omega \alpha}=0 .
\end{gathered}
$$

Solving the system of Equations (B8) and (B9) we find

$$
\begin{gathered}
\cos \theta=\frac{E_{J}}{S g^{2}}\left(1-\frac{\left\langle\hat{b}^{\dagger} \hat{b}\right\rangle}{S}\right)^{-1} \simeq \frac{E_{J}}{S g^{2}} \equiv \frac{g_{\mathrm{c}}^{2}}{g^{2}}, \\
\sqrt{\alpha}=-\frac{g S}{\sqrt{2 \omega}}\left(1-\frac{\left\langle\hat{b}^{\dagger} \hat{b}\right\rangle}{S}\right) \sin \theta \simeq \frac{g S}{\sqrt{2 \omega}} \sqrt{1-\frac{g_{\mathrm{c}}^{4}}{g^{4}}}
\end{gathered}
$$

where both the shift $\sqrt{\alpha}$ and rotation angle $\theta$ are nonzero when $g>g_{\mathrm{c}}$. Thus, using solutions (B10) and (B11) we obtain the initial Hamiltonian (B7) in a form similar to (B2), but renormalized with $\cos \theta$ coefficients:

$$
\begin{aligned}
\hat{H}= & \frac{E_{J} S}{2 \cos \theta}\left(1-\cos ^{2} \theta\right)-\frac{E_{J}}{\cos \theta}\left(S+\frac{1}{2}\right)+\omega\left(\hat{c}^{\dagger} \hat{c}+\frac{1}{2}\right) \\
& +\frac{E_{J}}{\cos \theta}\left(\hat{b}^{\dagger} \hat{b}+\frac{1}{2}\right)-\frac{g \cos \theta \sqrt{S \omega}}{2}\left(\hat{c}^{\dagger}-\hat{c}\right)\left(\hat{b}^{\dagger}-\hat{b}\right) .
\end{aligned}
$$

Next, we perform Bogoliubov's transformation that diagonalizes (B12), by performing a linear transform of Bose-operators $\hat{c}$ and $\hat{b}$ into Bose operators $\hat{e}_{1,2}$, and obtain

$$
\begin{aligned}
\hat{H}= & \frac{E_{J} S}{2 \cos \theta}\left(1-\cos ^{2} \theta\right)-\frac{E_{J}}{\cos \theta}\left(S+\frac{1}{2}\right)+\tilde{\varepsilon}_{1}\left(\frac{1}{2}+\hat{e}_{1}^{\dagger} \hat{e}_{1}\right) \\
& +\tilde{\varepsilon}_{2}\left(\frac{1}{2}+\hat{e}_{2}^{\dagger} \hat{e}_{2}\right)
\end{aligned}
$$

with the eigenvalues

$$
2 \tilde{\varepsilon}_{1,2}^{2}=\frac{E_{J}^{2}}{\cos ^{2} \theta}+\omega^{2} \pm \sqrt{\left(\frac{E_{J}^{2}}{\cos ^{2} \theta}-\omega^{2}\right)^{2}+4 \omega^{2} E_{J}^{2}}
$$

where both branches are now real for $g>g_{\mathrm{c}}=\sqrt{E_{J} / S}$ due to renormalization of the coefficients with $\cos \theta$ factors. We have expressed in (B14) the coupling constant $g$ via $\cos \theta$ using the self-consistency relation (B10). As is obvious from (B10) and (B11), both the shift $\sqrt{\alpha}$ and rotation angle $\theta$ progressively deviate from zero with increasing coupling strength $g$ in the interval $g>g_{\mathrm{c}}$, thus providing a description of the new stable phase of the system.

The ground-state energy of the system is now

$$
\widetilde{E}_{0}(S)=-\frac{E_{J}}{2 \cos \theta}(S+1)-\frac{E_{J} S}{2} \cos \theta+\frac{1}{2}\left(\tilde{\varepsilon}_{1}+\tilde{\varepsilon}_{2}\right),
$$

which always has a minimum at the end of the spin interval, at $S=N / 2$, thus justifying the Holstein-Primakoff approximation at finite angles $\theta$.

Thus, we found the second-order phase transition that is manifested by a gradual rotation of the total spin expectation value in the $y-z$ plane by an angle $\theta$ :

$$
\langle\hat{p}\rangle=-S g \sin \theta=\left\{\begin{array}{l}
0, \quad g<g_{\mathrm{c}} \\
\mp S g \sqrt{1-g_{\mathrm{c}}^{4} / g^{4}}, \quad g \geqslant g_{\mathrm{c}}
\end{array}\right.
$$

and

$$
\langle\hat{d}\rangle=2 e S l \sin \theta=\left\{\begin{array}{l}
0, \quad g<g_{\mathrm{c}} \\
\pm 2 e l S \sqrt{1-g_{\mathrm{c}}^{4} / g^{4}}, \quad g \geqslant g_{\mathrm{c}}
\end{array}\right.
$$

where $g_{\mathrm{c}}=\sqrt{2 E_{J} / N}$ and $S=N / 2$. The angle $\theta$ that describes the transition is plotted in Fig. 10.
[1] O. Viehmann, J. von Delft, and F. Marquardt, Superradiant Phase Transitions and the Standard Description of Circuit QED, Phys. Rev. Lett. 107, 113602 (2011).
[2] S. I. Mukhin and M. V. Fistul, Generation of non-classical photon states in superconducting quantum metamaterials, Supercond. Sci. Technol. 26, 084003 (2013). 
[3] M. A. Iontsev, S. I. Mukhin, and M. V. Fistul, Double-resonance response of a superconducting quantum metamaterial: Manifestation of nonclassical states of photons, Phys. Rev. B 94, 174510 (2016).

[4] M. Bamba, K. Inomata, and Y. Nakamura, Superradiant Phase Transition in a Superconducting Circuit in Thermal Equilibrium, Phys. Rev. Lett. 117, 173601 (2016).

[5] P. Nataf and C. Ciuti, No-go theorem for superradiant quantum phase transitions in cavity QED and counter-example in circuit QED, Nat. Commun. 1, 72 (2010).

[6] T. Jaako, Ze-Liang Xiang, Juan José Garcia-Ripoll, and P. Rabl, Ultrastrong-coupling phenomena beyond the Dicke model, Phys. Rev. A 94, 033850 (2016).

[7] A. Blais, R.-S. Huang, A. Wallraff, S. M. Girvin, and R. J. Schoelkopf, Cavity quantum electrodynamics for superconducting electrical circuits: An architecture for quantum computation, Phys. Rev. A 69, 062320 (2004).

[8] A. Wallraff, D. I. Schuster, A. Blais, L. Frunzio, R.-S. Huang, J. Majer, S. Kumar, S. M. Girvin, and R. J. Schoelkopf, Strong coupling of a single photon to a superconducting qubit using circuit quantum electrodynamics, Nature (London) 431, 162 (2004).

[9] J. M. Raimond, M. Brune, and S. Haroche, Manipulating quantum entanglement with atoms and photons in a cavity, Rev. Mod. Phys. 73, 565 (2001).

[10] L. DiCarlo, M. D. Reed, L. Sun, B. R. Johnson, J. M. Chow, J. M. Gambetta, L. Frunzio, S. M. Girvin, M. H. Devoret, and R. J. Schoelkopf, Preparation and measurement of three-qubit entanglement in a superconducting circuit, Nature (London) 467, 574 (2010).

[11] R. H. Dicke, Coherence in Spontaneous Radiation Processes, Phys. Rev. 93, 99 (1954).

[12] K. Rzażewski, K. Wódkiewicz, and W. Żakowicz, Phase Transitions, Two-Level Atoms, and the $\mathbf{A}^{2}$ Term, Phys. Rev. Lett. 35, 432 (1975)

[13] I. Bialynicki-Birula and K. Rzążewski, No-go theorem concerning the superradiant phase transition in atomic systems, Phys. Rev. A 19, 301 (1979).

[14] J. Keeling, Coulomb interactions, gauge invariance, and phase transitions of the Dicke model, J. Phys.: Condens. Matter 19, 295213 (2007)
[15] M. C. Cassidy, A. Bruno, S. Rubbert, M. Irfan, J. Kammhuber, R. N. Schouten, A. R. Akhmerov, and L. P. Kouwenhoven, Demonstration of an ac Josephson junction laser, Science 355, 939 (2017).

[16] H. Asai, S. Savel'ev, S. Kawabata, and A. M. Zagoskin, Effects of lasing in a one-dimensional quantum metamaterial, Phys. Rev. B 91, 134513 (2015).

[17] W. Kopylov, C. Emary, E. Schöll, and T. Brandes, Timedelayed feedback control of the Dicke-Hepp-Lieb superradiant quantum phase transition, New J. Phys 17, 013040 (2015).

[18] O. V. Astafiev, K. Inomata, A. O. Niskanen, T. Yamamoto, Yu. A. Pashkin, Y. Nakamura, and J. S. Tsai, Single artificial-atom lasing, Nature (London) 449, 588 (2007).

[19] S. Ashhab, J. R. Johansson, A. M. Zagoskin, and F. Nori, Singleartificial-atom lasing using a voltage-biased superconducting charge qubit, New J. Phys 11, 023030 (2009).

[20] S. André, V. Brosco, M. Marthaler, A. Shnirman, and G. Schön, Few-qubit lasing in circuit QED, Phys. Scr. T137, 014016 (2009).

[21] E. Almaas and D. Stroud, Dynamics of a Josephson array in a resonant cavity, Phys. Rev. B 65, 134502 (2002).

[22] W. A. Al-Saidi and D. Stroud, Eigenstates of a small Josephson junction coupled to a resonant cavity, Phys. Rev. B 65, 014512 (2001).

[23] C. Emary and T. Brandes, Chaos and the quantum phase transition in the Dicke model, Phys. Rev. E 67, 066203 (2003).

[24] N. Lambert, C. Emary, and T. Brandes, Entanglement and the Phase Transition in Single-Mode Superradiance, Phys. Rev. Lett. 92, 073602 (2004).

[25] A. Shnirman, G. Schön, and Ziv Hermon, Quantum Manipulations of Small Josephson Junctions, Phys. Rev. Lett. 79, 2371 (1997).

[26] T. Holstein and H. Primakoff, Field dependence of the intrinsic domain magnetization of a ferromagnet, Phys. Rev. 58, 1098 (1940).

[27] Y. Todorov, A. M. Andrews, R. Colombelli, S. De Liberato, C. Ciuti, P. Klang, G. Strasser, and C. Sirtori, Ultrastrong LightMatter Coupling Regime with Polariton Dots, Phys. Rev. Lett. 105, 196402 (2010). 